\title{
Mountain pastoralism in transition: Consequences of legalizing Cordyceps collection on yak farming practices in Bhutan
}

\author{
Kesang Wangchuk ${ }^{*}$ and Jigme Wangdi
}

\begin{abstract}
Yak farming is the main livelihood source for the high altitude communities in the eastern Himalaya. With increasing access to modern facilities, market opportunities and changes in the legal framework, pastoral systems in the Himalaya are undergoing an unprecedented change. Questionnaire-based qualitative surveys were conducted in five villages of northern Bhutan, to understand how the recent changes in the legal framework for Cordyceps (known as caterpillar fungus) collection have caused specific changes in yak farming practices. Survey results revealed that women were increasingly involved in yak husbandry and household work, after the legalization of Cordyceps collection in 2004. After legalization, the Cordyceps business overtook yak farming as the main income-earning activity. Post-legalization saw a decline in the overall grassland condition and most herders migrated a month earlier to the summer grazing land. Legalization also led to increase in the number of households buying commercial feeds for yaks. Yak mortality increased and fodder scarcity became more acute, which is a major constraint to yak farming. Despite the good income from the Cordyceps business, yak farming was the preferred earning activity over Cordyceps due to herders' confidence in yak farming as a reliable source of livelihood. Of several measures proposed by yak herders to improve yak farming, increasing grassland productivity and providing subsidies for feed purchases were the most important measures. The study concluded that yak farming practices have undergone a few positive but more undesirable changes after the legalization of Cordyceps collection in 2004. The results suggest multi-disciplinary approaches to address adequately the emerging issues of yak farming e.g. introducing schemes to make yak farming attractive to the mountain youth. The paper suggests interventions to strengthen yak farming and help herders make informed choices in the high altitude rangelands of Bhutan. Essentially, yak farming is at a crossroads where a firm decision is needed to either encourage and strengthen the farming practices or witness the gradual extinction of the age-old tradition.
\end{abstract}

Keywords: Gender; Grasslands; Himalaya; Rangelands; Caterpillar fungus

\section{Background}

Mountains occupy one fifth of the earth's surface and are home to at least 1.2 billion people (Körner and Ohsawa 2005). Inaccessibility and marginality make mountains one of the toughest environments for agriculture in terms of diversification of farm activities, resulting in a higher prevalence of vulnerability and warranting particular attention for sustainability of the overall mountain systems (Körner and Ohsawa 2005). In spite of the complex challenges, pastoralists adopt suitable strategies to sustain their livelihoods, with their ability to

\footnotetext{
* Correspondence: kesangwangchuk@rocketmail.com

Renewable Natural Resources Research Center, Ministry of Agriculture and Forests, Bumthang 32001, Bhutan
}

transform the extensive marginal rangelands into economically productive areas (Mishra et al. 2010). Pastoralists have sustained their economies through efficient utilization of grassland resources by high altitude livestock species, ranging from camelids in the Andes to yak (Bos grunniens) in the Himalayan mountains. However, pastoralism is evolving more rapidly due to changes in the socio-economic, institutional and policy environments (Shreshtha 1994). Several authors (Roder et al. 2002; Tulachan and Neupane 1999; Wangchuk et al. 2014) note that socio-economic development is the main driver of change in the Himalayan farming systems. To deepen our understanding of transitions in mountain farming in modern times, we investigated the case of 
yak farming in the Bhutan Himalaya, where farming practices have undergone an unprecedented change after the legalization of Cordyceps (Ophiocordyceps sinensis) collection for medicinal purposes.

Yak farming is predominant and a major livelihood source for the high altitude communities in Bhutan. However, recently yak farming has become marginalized and overtaken by the market demand for Cordyceps or the caterpillar fungus. Cordyceps, a restricted species until 2003, was legalized for collection in 2004, and has become a major source of income for the high altitude communities (Wangmo and Wangchuk 2008). Cordyceps are found in the remote northern parts of Bhutan, at an altitude of 3,400-4,100 $\mathrm{m}$ above sea level (Wangmo and Wangchuk 2008). The sale of Cordyceps fetches herding households a good annual income of up to US\$ 24,500 (Wangchuk et al. 2012). Internationally, the value of Cordyceps was reported to have increased by over 1,000\% between 1997 and 2012 (Winkler 2013). However, economic development has taken place in the mountains at a price. Environmental pollution through littering and grassland degradation are rampant (Wangchuk et al. 2012; Wangmo and Wangchuk 2008), and may consequently degrade the ecosystem services. Economic development in the mountains is also an indirect factor that weakens traditional cultures (Körner and Ohsawa 2005).

To date, empirical studies on Cordyceps have focused mainly on the medicinal properties (Boesi and Cardi 2009; Miller 2009; Panda and Swain 2011), sustainable management (Cannon et al. 2009; Tenzin 2009; Wangmo and Wangchuk 2008) and impacts on livelihood and environment (Wangchuk et al. 2012). A study investigating the effects of legalization of Cordyceps collection on yak farming practices was therefore needed. Wangmo and Wangchuk (2008) provide anecdotal evidence suggesting that Cordyceps collection may compete with yak farming, change yak farming practices and transform the lifestyle of herding communities. Despite these concerns, the changes remain unacknowledged and herding communities do not raise the issue for fear of inviting government policies banning the Cordyceps collection. Hence, there is little understanding of the long-term consequences of indiscriminate and unsustainable collection of Cordyceps to an age-old culture and farming environment of these rangeland communities.

Policies to promote livestock farming require sufficient knowledge of the existing production systems and changing practices, without which there could be highly diverse consequences on farm management and future evolution. Without this knowledge, it is highly likely that policy decisions will become difficult and impending conflicts more complex to resolve. Therefore, the objectives of this study were to understand how changes in the legal framework for Cordyceps collection have caused changes in yak farming practices in the high altitude rangelands of Bhutan, and to suggest future interventions for harmonious development of yak herding communities in view of Cordyceps collection activities. The paper unravels the consequences arising from Cordyceps collection activities, contributes to our understandings of the farming scenario and provides insights to policy decisions to benefit the yak herding communities in the longer run.

\section{Study areas}

The main Cordyceps collection areas are found in north eastern and western Bhutan. The villages of Naro, Lingzhi, SoiYaksa, Sephu and Khangdang were selected for the study (Figure 1).

The villages are far flung and about two days' walk from the nearest motor-able roads.

The topography of study villages is rugged and the mean maximum temperature of $20^{\circ} \mathrm{C}$ is recorded in summer and the mean minimum of $-10^{\circ} \mathrm{C}$ in winter. Summer is moderately warm and wet followed by freezing cold and dry winters. The annual rainfall shows a unimodal distribution pattern, with the maximum precipitation from June to September.

Every year, huge numbers of people spend about a month from mid-May to mid-June, collecting Cordyceps in the high altitude environment. Collection is banned in other months of the year. To prevent overexploitation of resource, the collection rules were revised within short intervals. Until 2007, only one person from a household was allowed to collect Cordyceps, however, in 2008, the revised rule allowed all members from a household to collect (Cannon et al. 2009; Gould 2007; Wangchuk 2008). From 2009 to date, a new rule was enforced, allowing only three persons from each household to collect. Many of the Cordyceps growing sites in Bhutan are near the border areas of Tibet. This makes easier for the people from the other side of the border to trespass and illegally collect Cordyceps in Bhutan. The practice continues unabated, even with the strict monitoring by the forestry and security personnel.

\section{Methods}

The study was conducted in the summer of 2014 in selected high altitude areas of Thimphu, Paro, Wangdue and Bumthang districts (Figure 1), where yak farming and Cordyceps collection are major income generating activities. For selecting villages and respondents, we employed the snowball sampling technique (Biernacki and Waldorf 1981) at two stages. In the first stage, researchers, development workers and local government bodies were consulted who provided a list of villages actively involved in Cordyceps business and yak farming. In the second stage, village leaders were consulted who 


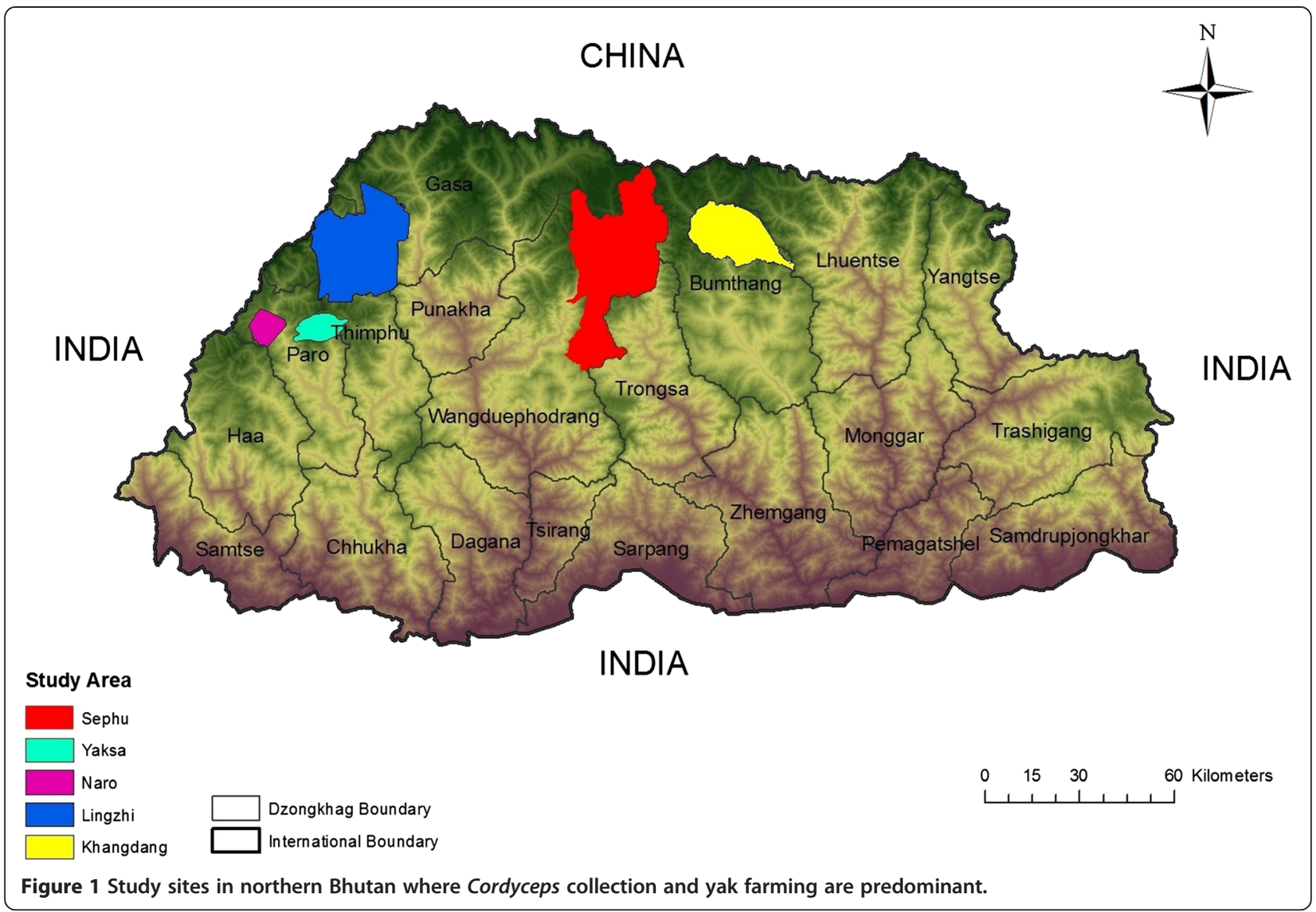

recommended households and elderly herders as potential respondents of survey interviews.

\section{Field interviews}

Using the snowball sampling technique (Biernacki and Waldorf 1981), a total of 100 older herders were identified, of who $60 \%$ were male. Both male (50-70 years of age) and female (50-65 years) herders from five villages were recruited as key informants since they were actively involved in yak farming over the last three decades and were able to provide farming details of the past, present and changes likely in the future. The selection criteria included: the herder engaged in yak farming for the past 30 years, have witnessed change in farming practices overtime, be a permanent resident of the village, and be willing to cooperate with the interviewer.

The survey was administered through questionnaires with open- and closed-ended questions. The survey questionnaire was divided into two parts. The first part consisted of questions on the overall situation 10 years before the legalization of Cordyceps collection. The same questions were repeated in the second part of the questionnaire in order to track changes in farming practices 10 years after the legalization. Information, missed by the questionnaire was captured through informal interviews and discussions during tea breaks and mealtimes after survey interviews. Topics and questions during informal discussions and interviews included hardships of yak farming, rural out-migration, incomes from yak farming and the Cordyceps business, and migratory and feeding practices for yaks. Informal discussions and interviews lasted from 30 minutes to one hour.

The first subpart of the questionnaire solicited information on the role of gender and socioeconomics of the herding community. The key informants were asked about the role of gender in yak husbandry. To better understand the changes in the population of the younger generation, the key informants were asked to provide opinions on population size before and after the legalization of Cordyceps collection. Questions were also asked to quantify changes in the number of households and herds. The key informants were requested to rank the income sources before and after the legalization of Cordyceps collection.

The second part of the questionnaire delved into grazing site condition and yak feeding practices. To simplify 
information, we used a three-point Likert scale (Likert 1932) against the commonly used five-point scale, since reliability and validity of information was found to be independent of the scale points employed by Likert-type items (Jacoby and Miichael 1971). The scale was used to gather information on whether the informants agreed, disagreed or did not know about the changes in grazing site condition, extent of forage oat cultivation and feeding of commercial animal feeds to yak, before and after the legalization of Cordyceps collection. We requested information on migratory practices, mainly on the time of migration to summer and winter grazing lands. This part of the questionnaire captured information on the yak mortality rate and its main causes, before and after the legalization of Cordyceps collection. The third part of the questionnaire included questions aimed at identifying the major constraints to yak farming. The constraints before and after the legalization of Cordyceps collection were compared.

The final part of the questionnaire sought general opinions on whether the herdsmen preferred yak farming or Cordyceps business. Accordingly, reasons were gathered to explain the preference for particular income-generating activities. The key informants were requested to opine on whether yak farming would decline in the future, followed by questions requesting to substantiate their opinions. Finally, the key informants were asked to put forward their important requests to the government, in order to improve and encourage yak farming in the high altitude rangeland.

\section{Data analysis}

Depending on the type of data, both parametric and nonparametric tests were performed. The pairedsamples $\mathrm{t}$ tests were performed on quantitative data. Opinions were displayed by percentages and the chisquare tests were conducted to evaluate differences in opinions before and after the legalization of Cordyceps collection. Ranks for income sources were analyzed with Wilcoxon signed-rank test. The entire dataset was analyzed with SPSS 22 (Landau and Everitt 2004). Comparisons were not made between villages since our interest was to understand the overall changes in yak farming practices before and after the legalization of Cordyceps collection.

\section{Results and discussion}

\section{Gender, livestock and socio-economics}

The percentage of family heads, yak herders by gender and the population size of younger generation, before and after the legalization of Cordyceps collection, are presented in Table 1. The results showed a significant decline in the proportion of men heading the family and herding yaks to and from the grazing fields, whereas the
Table $1 \mathrm{Gender}$ role and size of younger generation before (1993-2003) and after (2004-2014) the legalization of cordyceps collection

\begin{tabular}{|c|c|c|c|}
\hline & $\begin{array}{l}\text { Before legalizing } \\
\text { cordyceps collection (\%) } \\
\text { (Respondents } N=100 \text { ) }\end{array}$ & $\begin{array}{l}\text { After legalizing } \\
\text { cordyceps collection (\%) } \\
\text { (Respondents } N=100 \text { ) }\end{array}$ & Sig. \\
\hline \multicolumn{4}{|c|}{ Head of family } \\
\hline Men & 63 & 55 & $* *$ \\
\hline Women & 37 & 45 & $* *$ \\
\hline \multicolumn{4}{|l|}{ Yak herder } \\
\hline Men & 34 & 28 & * \\
\hline Women & 57 & 62 & * \\
\hline Children & 9 & 10 & ns \\
\hline \multicolumn{4}{|c|}{ Population size of younger generation } \\
\hline Small & 30 & 62 & ** \\
\hline Medium & 25 & 14 & ** \\
\hline Big & 45 & 14 & $* *$ \\
\hline $\begin{array}{l}\text { Number of } \\
\text { households }\end{array}$ & 837 & 989 & ns \\
\hline $\begin{array}{l}\text { Number of } \\
\text { herds }\end{array}$ & 591 & 516 & ns \\
\hline
\end{tabular}

${ }^{*} \mathrm{p} \leq 0.05,{ }^{* *} \mathrm{p} \leq 0.01$, ns-non-significant.

proportion of women as head of family and involvement in yak herding increased significantly after the legalization of Cordyceps collection. The proportion of family heads and yak herders differed by gender, which is consistent with the finding of Joshi et al. (2013) that women are increasingly involved in rangeland resource management in the Hindu Kush Himalayan region. Following legalization of Cordyceps collection, women are being increasingly involved in animal husbandry and household work, suggesting the growing leadership of women in domestic affairs. This is a positive development, indicating considerable improvement in women's role at the family level, suggesting that women might have more control over yak farming in the future. It would also mean that such positive developments may overcome gender-based constraints hindering women as farmers and managers of natural resources, particularly from the perspective of the future of yak farming. However, the bigger role of women would also mean increase in responsibilities and labour burden of women. Although, this study was unable to generate evidence on how men devote most of their time to off-farm activities such as Cordyceps collection, tourism and market related activities, Joshi et al. (2013) reported that men's roles in the Himalayan region are limited to their involvement in the wage labour and marketing of agricultural products and they spend less time on pastoral activities, as compared with women. Therefore, the results suggest that 
men shall have a greater role in off-farm income generating activities in the future. The findings of this study on the changing role of gender should not be considered as a general trend but mainly a phenomenon in yak farming and Cordyceps growing regions in the Bhutan Himalaya.

The population size of the younger generation was significantly smaller after the legalization of Cordyceps collection (Table 1), showing a decline in the population of rural youth. This is likely to result in acute shortage of farm labourers and may impede yak farming in the future. Informal discussions with both young and old villagers revealed that yak farming is becoming increasingly difficult. The younger generation are aware of the hardship of yak husbandry in remote and inaccessible areas and the low return on investment as compared to the off-farm businesses such as tourism and Cordyceps collection. Thus, yak farming is becoming less popular with the younger generation. Although, the causes of rural youth decline are multiple and complex, Wangchuk et al. (2014) attributed rural-urban migration to the rising tendency of young and literate people to migrate to urban areas seeking better economic opportunities. Relating the current trend of declining number of youth to the future population structure, our study suggests that the future herding community will most likely be comprised of elderly people. This cautions the need for the herding communities to adapt to the generational change. Accordingly, it sends out an important signal for the government agencies to step up efforts to address the issue, if yak farming is not to face extinction.

Although non-significant, we detected an increase in the number of households by 152 over the last two decades and a decline in the number of herds by 75 after Cordyceps collection (Table 1). The increase in household numbers and decline in herd numbers may not necessarily be due to legalization but can be attributed to two other possible reasons. Firstly, the increase in household numbers is probably attributable to the customary practice of traditional rural families in Bhutan of dividing land between the family members, with each generation inheriting consecutively smaller patches of land (Wangchuk et al. 2014). The new houses built on inherited land results in the increase in number of households over the years. Secondly, informal discussions revealed that, due to better economic opportunities elsewhere and increasing difficulty in yak farming, there are cases of some herding families giving up yak farming and migrating to urban areas in the recent years. The herds were often sold to fellow herders, which might explain the consequent decline in the total number of herds without a decline in overall yak numbers, while individual herd sizes may have increased.
About 77 percent of respondents ranked yak farming as the main source of income for the herding families before legalizing collection of Cordyceps (Figure 2). However, after 2004, with herding families legally authorized to collect Cordyceps, about 70 percent of respondents mentioned the collection and sale of Cordyceps as the main source of income, followed by yak farming. Herdsmen often claim that the income obtained from a month- long collection of Cordyceps is by many folds greater than the income earned from year-long yak farming. This is probably the main reason why the numbers of Cordyceps collectors are on the rise in the recent years (Wangchuk et al. 2012).

\section{Grassland condition}

Grazing land was said to have degraded after the legalization of Cordyceps collection, according to over 70 percent of the respondents (Figure 3). The results highlight the perception of increased degradation of the grasslands arising from continued intensive collection of Cordyceps and overgrazing by yaks and horses. Amongst the causes of grazing land degradation, Cordyceps collection activity was stated as the main cause by over 50 percent of respondents. According to herdsmen during the informal discussions, the number of illegal collectors from across the Bhutan-Tibet border is unaccounted for, and was said to outnumber the Bhutanese collectors, which contributes to grazing land degradation. Wangmo and Wangchuk (2008) reported that Cordyceps collection involved digging up soil, which is often left unattended, exposing the bare soil to erosion by monsoonal rain. Similarly to findings by Wangchuk et al. (2012), key informants mentioned littering of non-degradable plastic food wrappers and bottles by Cordyceps collectors and lack of proper garbage management as one of the causes of environmental degradation.

According to a quarter of respondents, overgrazing is another cause of grassland degradation. Herders mentioned that, the Cordyceps collection has led to an increase in the number of horses grazing the grasslands. With the rising number of collectors over recent years, a large number of horses are used to transport basic necessities to the collection sites in order to support a month-long collection of Cordyceps in a difficult terrain. Furthermore, in the informal discussions, the herdsmen described the situation being aggravated by grazing pressure from horses used for tourism activities. Wangchuk et al. (2006) equated grazing by one horse to grazing by ten adult cattle for one day in the same area. Further, the carrying capacity of high altitude grassland is low, with one livestock unit requiring about 4.5 ha of well-managed grassland (Wangchuk et al. 2013). Thus, overstocking on less productive grassland explains the overgrazing and consequent degradation of grassland. 


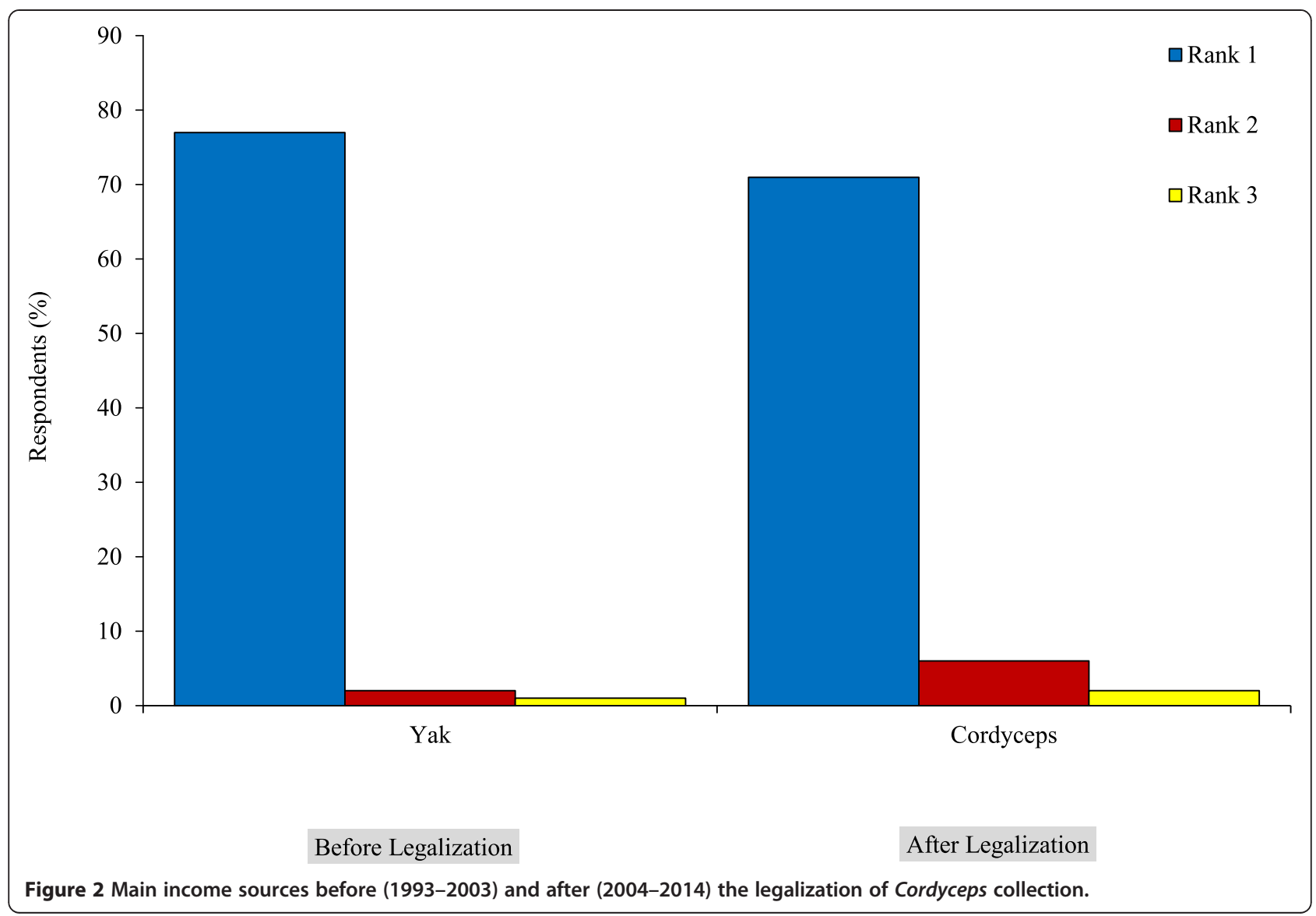

\section{Herding practices and constraints to yak farming}

The yak herders migrate to summer grazing pastures in April and May, located in the high altitude region between 3,800-4,500 $\mathrm{m}$ above sea level; these are also the sites for Cordyceps collection. Although, there was no significant change in timing of migration to the winter grazing land, we detected a significant difference in migration timing to the summer grazing land, before and after the legalization of Cordyceps collection (Figure 4). The proportion of herders migrating to the summer grazing land in April increased by about 20 percent after the legalization. On the contrary, we detected a significant drop of about 25 percent in the proportion of herders migrating to the summer grazing land in May. The shift in time of migration suggests that most herders are spending more time in the summer grazing land, after the legalization. Since herders found that summer grazing lands are increasingly degraded by Cordyceps collection activities with consequent decrease in the productivity of grasslands, to ensure sufficient forage for their yaks, most herders migrate to the summer grazing land a month earlier, after legalization of Cordyceps collection, as opposed to the migration time followed before legalization.
Our study showed improvements in yak feeding practices after the legalization. We detected a fivefold increase in the proportion of respondents feeding commercial feed (oil cakes and pellets) to yaks (Table 2). This practice reflects the greater purchasing power from the Cordyceps business, which was the main income source after the legalization. This is a positive trend, indicating that yaks are receiving better nutrition. However, about 77 percent of respondents agreed that yak mortality has increased after the legalization. Of the several reasons outlined, forage scarcity featured as the most important cause of yak mortality and a major constraint to yak farming, followed by wildlife predation and disease (Coenurus multiceps multiceps) (Table 2). This means that legalization and escalating pasture forage shortages have contributed to the deteriorating situation of yak farming in the Bhutan Himalaya.

\section{Farming preference and future of yak farming}

About 63 percent of respondents preferred yak farming over the Cordyceps business in the long term (Table 3). This finding suggests that yak farming is an assured source of steady income for a large number of respondents, in spite of the Cordyceps being a lucrative business 


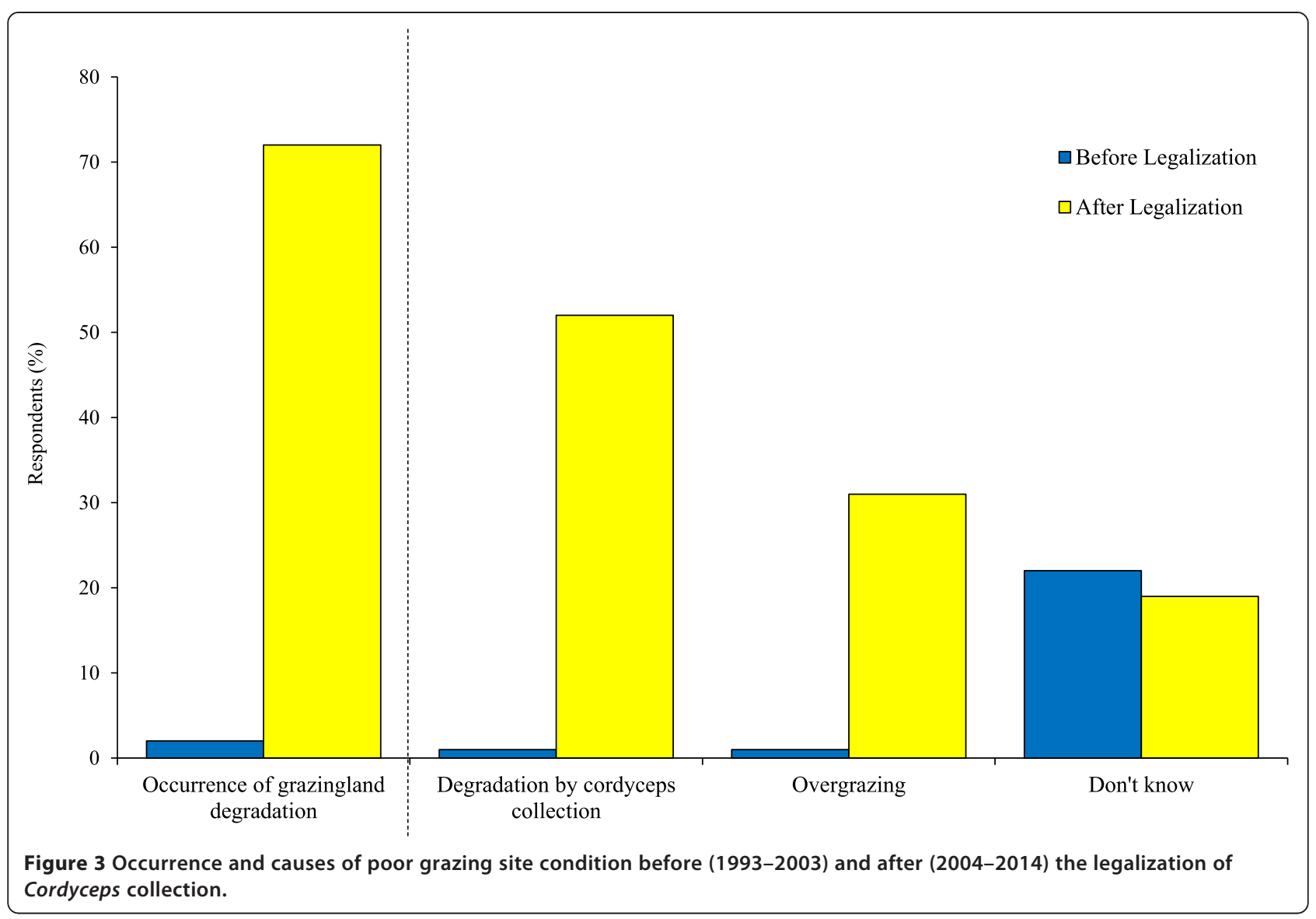

venture. When probed on the main reason for respondents' preferences, yak farming was mentioned to be sustainable and resilient at the time of food scarcity. Yaks have been reared for centuries and are strongly linked to the herders' identity and culture, which instils more confidence for yak farming. The yaks survive the harsh conditions and the herders have an intuitive ability to sustainably derive their livelihood from yak farming (Khan 1995). On the contrary, most respondents viewed Cordyceps collection as an uncertain future, due to overexploitation of the resource, fluctuation in market price and greater risks involved in collection activities in the difficult terrain under harsh weather conditions. This reiterates the prediction of Wangchuk et al. (2006) that the price of Cordyceps would fluctuate in the future. Cordyceps, a resource of immense commercial value, runs the risk of over-exploitation and the issue of sustainability looms large (Winkler 2013). Reports from some parts of the Cordyceps growing region in China indicate a sharp decline in Cordyceps production due to over-harvesting (Stone 2008; Winkler 2013). Winkler (2013) fears that the overly intense collection could lead to extinction of the Cordyceps resource. In Bhutan, without adequate monitoring of the Cordyceps sites, the illegal harvesting by the poachers from across the border with Tibet will threaten the sustainability of the resource.

Despite the fact that yak farming is preferred over the Cordyceps business, about 88 percent of respondents felt that yak farming will decline in the future (Table 3). This was linked to yak farming becoming difficult, mainly because of the increased forage shortage and yak mortality due to disease. The second important reason put forward for the likely decline in yak farming was rural out-migration. According to respondents during the informal discussions, the migration trend is on the rise and the younger generation seemed to show less interest to take up yak farming. Rural-urban migration is not only a Bhutanese phenomenon; it has been reported in most rural regions around the world due to better economic opportunities elsewhere, causing damage to rural communities in terms of skewed demographic profiles, reductions in services and loss of local culture (Stockdale 2004). Rural out-migration is becoming common in most Asian countries, where the exit of younger family members is leaving more labour burdens on elderly people and children in rural areas, leading to rise in child farm 


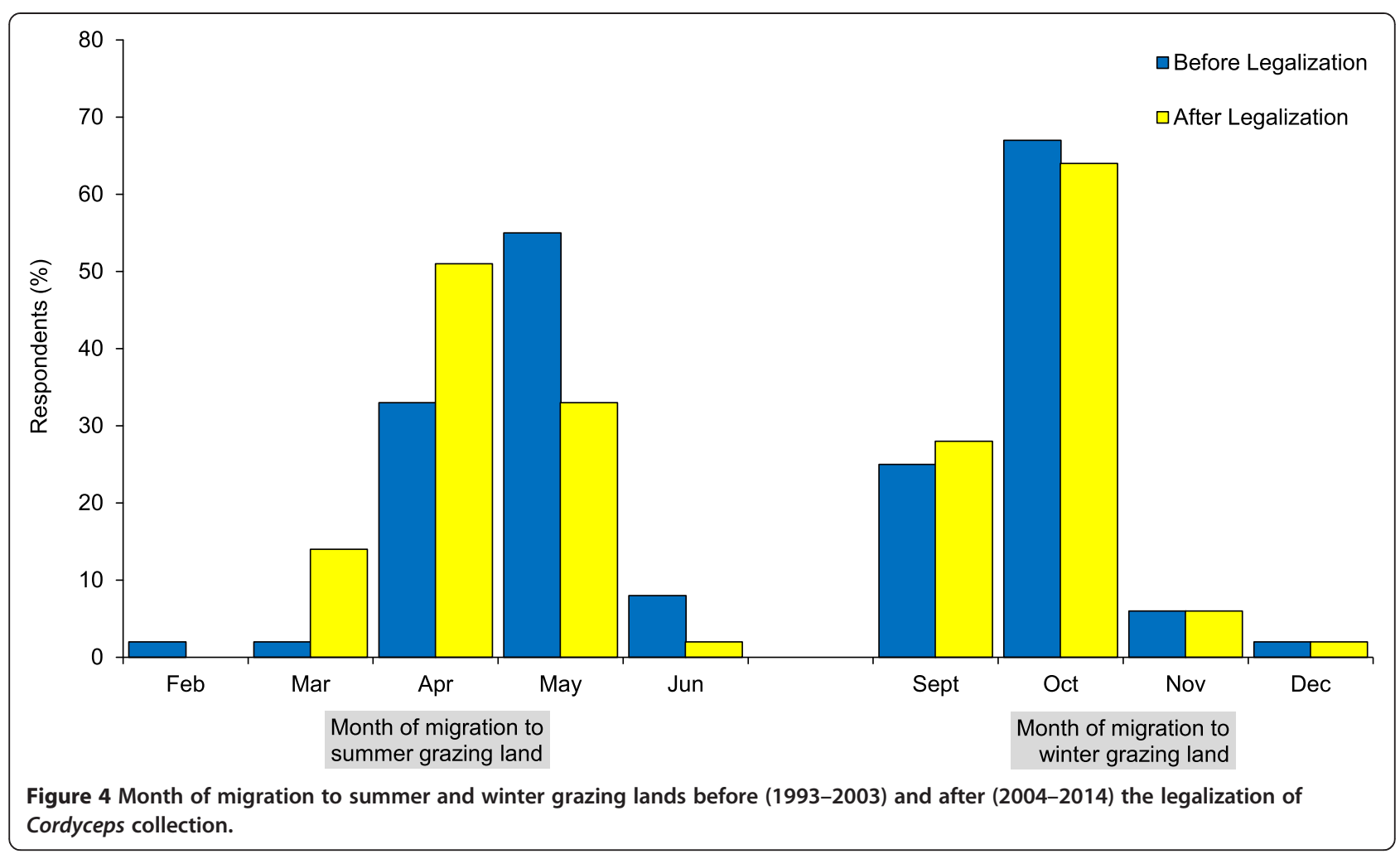

Table 2 Yak mortality, feeding practice, causes of yak mortality and constraints to yak farming before (1993-2003) and after (2004-2014) the legalization of cordyceps collection

\begin{tabular}{|c|c|c|c|}
\hline & \multicolumn{2}{|l|}{ Respondents (\%) } & \multirow[b]{2}{*}{ Sig. } \\
\hline & $\begin{array}{l}\text { Before legalizing } \\
\text { cordyceps collection }\end{array}$ & $\begin{array}{l}\text { After legalizating } \\
\text { cordyceps collection }\end{array}$ & \\
\hline $\begin{array}{l}\text { 1. Commercial feeds } \\
\text { fed to yaks }\end{array}$ & 20 & 80 & $* * *$ \\
\hline 2. Yak mortality & 68 & 77 & * \\
\hline \multicolumn{4}{|l|}{ Causes of yak mortality } \\
\hline 1. Forage shortage & 30 & 34 & ns \\
\hline 2. Wildlife attack & 18 & 20 & ns \\
\hline 3. Gid disease & 11 & 14 & ns \\
\hline 4. Accidents & 11 & 10 & ns \\
\hline 5. Don't know & 26 & 21 & ns \\
\hline \multicolumn{4}{|c|}{ Constraints to yak farming } \\
\hline 1. Forage shortage & 68 & 77 & * \\
\hline 2. Wild life attack & 21 & 19 & ns \\
\hline 3. Gid disease & 7 & 7 & ns \\
\hline 4. Don't know & 5 & 3 & ns \\
\hline
\end{tabular}

${ }^{*} p \leq 0.05,{ }^{* * *} p \leq 0.001$, ns-non-significant. labour (Meerza 2010). The present scenario highlights the need to consider introducing better rural schemes and opportunities to retain rural youth and encourage yak farming. The growing interest in Cordyceps business shall also contribute to a decline in yak farming. This is because the income from the sale of Cordyceps is invested in land and infrastructure in the urban areas, where the herdsmen and their families are spending more time per year.

\section{Future interventions}

The measures proposed by respondents to improve and encourage yak farming are presented in Table 4. Over half of the respondents felt that the improvement in yak farming can be possible through an increase in forage production, in terms of both quantity and quality, at subsidized rates. Improvements in forage production and availability may be beneficial for yak enterprises, but our study shows that the viability of yak farming will be threatened by the increasing farm labour shortage resulting from rural out-migration.

The need to improve the breed of yaks was the second most important request, by $15 \%$ herders who thought the yak breed is on the decline. More importance placed on the Cordyceps business could probably have led to less attention on improving yak breed. The decline in breed could also be explained by the repeated and traditional 
Table 3 Main income source, preference for income activities and opinions on future yak farming

\begin{tabular}{|c|c|c|}
\hline Survey question & Herders' opinion & Respondents (\%) \\
\hline \multirow{2}{*}{$\begin{array}{l}\text { Which income } \\
\text { generating activity } \\
\text { would you prefer } \\
\text { in the long run? }\end{array}$} & 1. Yak farming & 63 \\
\hline & 2. Cordyceps collection & 37 \\
\hline \multirow[t]{2}{*}{$\begin{array}{l}\text { Why do you prefer } \\
\text { the particular activity? }\end{array}$} & $\begin{array}{l}\text { 1. Yak farming is sustainable } \\
\text { and Cordyceps has } \\
\text { uncertain future }\end{array}$ & 63 \\
\hline & 2. Cordyceps is sustainable & 37 \\
\hline \multirow{3}{*}{$\begin{array}{l}\text { Will the yak farming } \\
\text { decline in future? }\end{array}$} & 1. Yak farming will decline & 88 \\
\hline & 2. Yak farming will not decline & 11 \\
\hline & 3. Don't know & 01 \\
\hline \multirow{5}{*}{$\begin{array}{l}\text { What are the main } \\
\text { reasons likely to cause } \\
\text { decline in yak farming } \\
\text { in future? }\end{array}$} & $\begin{array}{l}\text { 1. Yak farming is getting } \\
\text { difficult }\end{array}$ & 37 \\
\hline & 2. Rural-urban migration & 31 \\
\hline & 3. Cordyceps collection & 21 \\
\hline & 4. Wildlife attack & 06 \\
\hline & 5. Don't know & 05 \\
\hline
\end{tabular}

pure breeding practices common in the yak herding community which are reported to have harmful effects on yak performance (Wiener et al. 2003). Inbreeding in yaks is a particular problem in Bhutan, Nepal and India (Cai and Wiener 1995) and the lack of an efficient yak breeding program to maintain the genetic diversity in yaks is reported as a major problem in Nepal (Shaha 2000).

The need for rules and regulations to prevent degradation of grazing land by Cordyceps collection activities was the third most important request to the government. The high altitude communities are fully aware of the ill effects of Cordyceps collection on grazing land and they expressed concern to prevent decline in grazing land productivity. Our findings confirm the

Table 4 Government interventions requested by herding communities to sustain yak farming

\begin{tabular}{lll}
\hline SI. no. & $\begin{array}{l}\text { Measures requested by respondents to } \\
\text { improve yak farming }\end{array}$ & Respondents (\%) \\
\hline 1 & $\begin{array}{l}\text { Must increase forage production and } \\
\text { make commercial feeds available at } \\
\text { subsidized rate }\end{array}$ & 50 \\
\hline & $\begin{array}{l}\text { Must improve quality of breed of yak } \\
3\end{array}$ & 15 \\
Must introduce regulations to prevent & 13 \\
& $\begin{array}{l}\text { grazing land degradation by Cordyceps } \\
\text { collection activities }\end{array}$ & \\
Must provide effective and timely & 11 \\
5 & veterinary services to control gid disease & 09 \\
6 & Other minor reasons & 02 \\
\hline
\end{tabular}

apprehension of Wangmo and Wangchuk (2008) who found the collection techniques used by Cordyceps collectors destructive to grasslands.

\section{Conclusions}

Our study highlights a series of changes in yak farming practices after the legalization of Cordyceps collection. Legalization has resulted in a few positive but more undesirable changes in overall yak farming. Positive changes included more income from Cordyceps business and better nutrition for yaks. Undesirable changes were the increased role of women in household chores, deteriorated grassland condition, and increased yak mortality due to acute pasture shortage. Legislation has partly contributed to rural-urban migration. The escalating competition between yak farming and Cordyceps business may lead to a decline in yak farming in the long run. This may accelerate the rural out-migration and aggravate the farming situation in the mountains. Yak farming is becoming increasingly vulnerable as the population of younger rural people is reduced and productivity of grasslands declines. Although difficult to achieve, appropriate schemes are needed to make yak farming attractive to the mountain youth. The high altitude communities shall continue to benefit substantially from the Cordyceps business. However, it is of paramount importance to invent and promote suitable harvesting techniques to ensure minimum damage to the grassland. It is hoped that the future government interventions will focus on increasing the productivity of grasslands, improving the breed of yaks and regulating Cordyceps collection to avoid grassland degradation. The government also needs to put in place a levy mechanism, whereby a small percentage of income from Cordyceps is invested in grassland renovation. In essence, yak farming is at a crossroads where a firm decision is needed to either strengthen the age-old tradition or witness its gradual extinction.

\section{Competing interests}

The authors declare that they have no competing interests.

\section{Authors' contributions}

Both authors contributed to development of the concept and survey questionnaire, field interviews and data collection, data analysis and drafting of the manuscript. The final manuscript was approved by both authors.

\section{Authors' information}

KW (Ph.D.) is Pasture Ecologist and Deputy Chief Research Officer at the Renewable Natural Resources Research and Development Center (RNRRDC) in Bumthang, Bhutan and carries out research on rural livelihood, poverty alleviation, climate change and grasslands. JW is a Principal Livestock Health Officer at RNRRDC, Bumthang and conducts research on poverty alleviation and rural dairy development.

\section{Acknowledgements}

The authors gratefully acknowledge the financial support of the Royal Government of Bhutan. We also thank Mr. Thubten, Mr. Samten Nidup, Mr. Harilal Nirola and Mr. Karma Dorji for their valuable assistance during the field surveys. 
Received: 27 October 2014 Accepted: 27 January 2015

Published online: 10 February 2015

\section{References}

Biernacki, Patrick, and Dan Waldorf. 1981. Snowball sampling. Sociological Methods and Research 10: 141-163.

Boesi, Alessandro, and Francesca Cardi. 2009. Cordyceps sinensis medicinal fungus: traditional use among tibetan people, harvesting techniques, and modern uses. Herbalgram 83: 52-63.

Cai, Li, and Gerald Wiener. 1995. The Yak. Bangkok: Thailand Food and Agricultural Organization of the United Nations.

Cannon, PF, NL Hywel-Jones, N Maczey, Lungten Norbu, Tashi Samdrup Tshitila, and Phuntso Lhundup. 2009. Steps towards sustainable harvest of Ophiocordyceps sinensis in Bhutan. Biodiversity Conservation 18: 2263-2281.

Gould, Rachelle. 2007. Himalayan Viagra, Himalayan gold? Cordyceps sinensis brings new forces to the Bhutanese Himalaya. Tropical Resource Bulletin 26: 63-69.

Jacoby, Jacob, and Matell Miichael. 1971. Three-point Likert Scales are Good Enough. Journal of Marketing Research 8: 495-500.

Joshi, Srijana, RM Shrestha, Dechen Sherpa, Binay Shrestha, Yin Lun, Yeshey Dorji, Gan Tingyu, Ni Jioubing, Jun lai, Yangzong Ciren, Khadka Manohara, and Verma Ritu. 2013. Gender and Pastoralism in the Rangelands of the Hindu Kush Himalayas: From the margins to new heights. Kathmandu, Nepal: International Centre for Integrated Mountain Development.

Khan, Obaidullah. 1995. Forward speech. In The yak, Bangkok, Thailand.: FAO (Food and Agricultural Organization of the United Nations) Regional Office for Asia and the Pacific.

Körner, Christian, and Masahiko Ohsawa. 2005. Mountain Systems. In Ecosystems and Human Well-being: Current State and Trends. Millennium Ecosystem Assessment, Washington, DC: Island Press 681-716.

Landau, Sabine, and BS Everitt. 2004. A Handbook of Statistical Analyses Using SPSS. Boca Raton: FL Chapman \& Hall/CRC.

Likert, Rensis. 1932. A Technique for the Measurement of Attitudes. Archives of Psychology 140: 1-55.

Meerza, SIA. 2010. Rural-urban Migration and Its Consequences on Rural Children: An Empirical Study. Asian Social Science 6: 176-181.

Miller, RA. 2009. The Cordyceps sinensis medicinal mushroom. www.nexusmagazine. $\mathrm{com} /$ products/the-cordyceps-sinensis-medicinal-mushroom-detail. Accesed 20 September, 2014

Mishra, Charudutt, Bagchi Samanta, Namgail Tsewang, and YV Bhatnagar. 2010. Multiple Use of Trans-Himalayan Rangelands: Reconciling Human Livelihoods with Wildlife Conservation. In Wild rangelands: Conserving Wildlife While Maintaining Livestock in Semi-arid Ecosystems, 291-311. Chichester: Wiley-Blackwell.

Panda, AK, and KC Swain. 2011. Traditional uses and medicinal potential of Cordyceps sinensis of Sikkim. Journal of Ayurveda Integrated Medicine 2: 9-13.

Roder, Walter, Georg Gratzer, and Kinzang Wangdi. 2002. Cattle grazing in the conifer forests of Bhutan. Mountain Research and Development 22: 1-7.

Shaha, BKP. 2000. Economics of yak farming with relation to tourism in Nepal The third international congress on yak. Lhasa, China. Kenya: International Livestock Research Institute.

Shreshtha, Surendra. 1994. Evolution of mountain farming systems. Proceedings of the FAO/ICIMOD seminar at Lumle Agricultural Research Center. 13-29. Kathmandu, Nepal.

Stockdale, Aileen. 2004. Rural out-migration: community consequences and individual migrant experiences. Sociologia Ruralis 44: 167-194.

Stone, Richard. 2008. Last stand for the body snatcher of the Himalayas? Science 322: 1182.

Tenzin, K. 2009. Current Harvesting and Management Practices for Yartsa guenboob in Bhutan Regional Conference on Cordyceps Management and Marketing Bumthang. Bhutan: Ministry of Agriculture.

Tulachan, PM, and A Neupane. 1999. Livestock in Mixed Farming Systems of the Hindu Kush-Himalayas: Trends and Sustainability. Kathmandu: Nepal International Centre for Integrated Mountain Development.

Wangchuk, Dorji. 2008. Cordyceps sinensis: Is Domestication Possible? In Kuensel: Bhutan's National Newspaper.

Wangchuk, Kesang, Karma Dorii, and Ugyen Lhendup. 2006. Crushing the Bone: Minimizing Grazing Conflicts in Community Tsamdro- A Case Study from Dhur Village. Bumthang, Bhutan: Choekhor Geog.

Wangchuk, Kesang, Maria Wurzinger, Andras Darabant, Georg Gratzer, and Werner Zollitsch. 2014. The changing face of cattle raising and forest grazing in the Bhutan Himalaya. Mountain Research and Development 34: 131-138.
Wangchuk, Kesang, Tshering Gyaltshen, Thrimshing Yonten, Harilal Nirola, and Nidup Tshering. 2013. Shrubland or Pasture? Restoration of Degraded Meadows in the Mountains of Bhutan. Mountain Research and Development 33: 161-169.

Wangchuk, Sangay, Norbu Nawang, and Sherub. 2012. Impacts of Cordyceps Collection on Livelihoods and Alpine Ecosystems in Bhutan as Ascertained from Questionnaire Survey of Cordyceps Collectors. Bumthang Royal Government of Bhutan: UWICE Press.

Wangmo, Sonam, and Kesang Wangchuk. 2008. The collectors' dilemma: Constraints and opportunities for community based Cordyceps management in Lingzhi. Journal of Natural Resources Bhutan 4: 172-174.

Wiener, G.Jianlin, H. and Ruijun, L. 2003. Breeding, Crossbreeding and Hybridizing in Yak. In Bangkok, Thailand, 33-35. Food and Agriculture Organization of the United Nations, Regional Office for Asia and the Pacific.

Winkler, Daniel. 2013. Steps towards Sustainable Harvest of Yartsa Gunbu (Caterpillar Fungus, Ophiocordyceps sinensis) Proceedings of the 7th International Medicinal Mushroom Conference. Beijing 635-644.

\section{Submit your manuscript to a SpringerOpen ${ }^{\mathcal{D}}$ journal and benefit from:}

- Convenient online submission

- Rigorous peer review

- Immediate publication on acceptance

- Open access: articles freely available online

High visibility within the field

- Retaining the copyright to your article

Submit your next manuscript at $\gg$ springeropen.com 